\title{
Um Programa de Computador para Simular Alguns Aspectos Operacionais da Soldagem MIG/MAG
}

\author{
(A Piece of Software to Simulate Operational Characteristics of MIG/MAG Welding)
}

\author{
Paulo J. Modenesi ${ }^{1}$, Sandro Matilde ${ }^{l}$, Gabriel Lacerda Q. Cançado' ${ }^{2}$ Carlos Eduardo Duarte de Melo ${ }^{1}$ \\ ${ }^{1}$ Universidade Federal de Minas Gerais, Belo Horizonte, MG, Brasil, modenesi@demet.ufing.br
}

\begin{abstract}
Resumo
A soldagem MIG/MAG e processos correlatos, que usam o arco e a alimentação contínua de arame, permanecem como os de maior importância industrial para a união de peças metálicas. Estes processos tendem a apresentar um elevado número de variáveis. A soldagem MIG/MAG é, ainda, sujeita a diferentes fenômenos que podem causar dificuldades durante a sua aplicação e, em particular, no planejamento e na definição das condições operacionais para uma dada aplicação. O presente trabalho descreve o desenvolvimento de um modelo para simular a variação de algumas variáveis do processo (como a corrente e tensão de soldagem e o comprimento do arco) e a sua implementação em um programa de computador. As equações básicas do modelo são apresentadas e discutidas com considerações sobre a sua aplicação. Resultados de testes experimentais para a determinação de alguns parâmetros fundamentais do modelo são também apresentados e discutidos. Simulações usando o programa desenvolvido são apresentadas juntamente e comparadas com testes reais de soldagem. Ferramentas do programa para simular mudanças nas condições operacionais de soldagem, no comportamento do arco elétrico e na operação da fonte de soldagem são apresentadas. Encaminhamentos baseados em abordagem teórica e empírica para algumas destas capacidades são apresentados.
\end{abstract}

Palavras-chave: GMAW; Modelagem; Parâmetros; Simulação.

Abstract: $M I G / M A G$ welding and other processes that are based on arc heating and the feeding of a continuous wire electrode are still the most used metal joining processes in the industry. These processes present a large number of variables. Furthermore, MIG/MAG welding is subjected to several phenomena that cause unexpected changes in parameters and make it difficult to predict operational conditions for a given job. The paper describes the development and the software implementation of a mathematical model to simulate some operational aspects of the MIG/MAG welding process. The basic equations of the model are presented and discussed. Simulations of different welding conditions are presented and compared to results from actual welding tests. Software tools to simulate alterations in welding conditions, in arc behavior, and power supply operation are also described. Approaches to these tools, based on both theoretical and empirical considerations, are presented.

Key-words: GMAW; Modeling; Welding Parameters; Simulation.

\section{Introdução}

A soldagem MIG/MAG é atualmente o mais importante processo industrial para a união de ligas metálicas, particularmente os aços. Este processo apresenta diversas vantagens em relação ao processo de soldagem com eletrodos revestidos, com destaque à sua possibilidade de ser mecanizada ou automatizada de forma relativamente fácil, a sua maior produtividade e maior facilidade de uso. A soldagem MIG/MAG, juntamente com a soldagem com arames tubulares é amplamente utilizada em diferentes ramos da indústria metal-mecânica com destaque para a indústria automobilística, de autopeças e de fabricação de estruturas. Apesar de suas vantagens, o processo MIG/MAG apresenta, quando comparado com a soldagem com eletrodos revestidos e outros processos, uma maior dificuldade de ser

(Recebido em 29/02/2012; Texto final em 01/03/2012).

Artigo originalmente publicado no CONSOLDA 2011 ajustado, em parte devido à sua elevada sensibilidade a variações de seus parâmetros operacionais. Neste sentido, um grande número de fatores como, por exemplo, a composição química do eletrodo e seu diâmetro, a composição do gás de proteção, a configuração geométrica do sistema, as características do metal de base e as características do equipamento podem afetar a operação. Esta sensibilidade, em conjunto com diversos fatores externos, incluindo a condição do equipamento de soldagem, pode gerar situações de operação instável, de difícil controle e, muitas vezes, de difícil diagnóstico.

O desenvolvimento de modelos matemáticos para simular o comportamento deste processo (e de outros processos de soldagem em geral) é uma alternativa interessante para prever o comportamento do processo, reduzir o número de testes de soldagem para o ajuste das condições de operação e estudar diversos fenômenos importantes do processo.

Esta modelagem foi feita inicialmente diretamente a partir de resultados experimentais usando diferentes técnicas estatísticas, sendo esta técnica ainda amplamente usada atualmente para descrever diferentes aspectos do processo com destaque para o 
formato do cordão de solda. Como exemplos de trabalhos que se enquadram nesta linha, podem ser citados os trabalhos de Kim [12], Murugana [3], Quiang [4] e Palani [5]. Técnicas alternativas similares, particularmente com o uso de Redes Neurais Artificiais, também têm sido usadas para simular a geometria do cordão de solda [6-7] e outros aspectos do processo. Todas estas técnicas dependem fundamentalmente das condições dos testes realizados para levantar os dados usados na modelagem, não sendo usados princípios fundamentais do processo e, neste sentido, os modelos resultantes podem ser considerados como do tipo "caixa preta". Embora interessantes para representar e otimizar diferentes aspectos de uma operação de soldagem, este tipo de modelo, em geral, não fornece indicações para as causas ou mecanismos responsáveis pelos fenômenos observados e devem ser usados com extremo cuidado para analisar resultados obtidos com condições operacionais mesmo que apenas ligeiramente diferentes.

Possivelmente, as técnicas de modelagem mais sofisticadas e cada vez mais usadas atualmente envolvem o uso de ferramentas numéricas de discretização para a solução de equações diferenciais representativas de diferentes aspectos do processo em estudo. Estas técnicas foram usadas para estudar fenômenos no arco e na poça de fusão [8-14] e a dissipação de calor na peça [15-16].

Uma terceira alternativa envolve a solução analítica ou numérica de modelos teóricos simplificados. Em particular, a variação da corrente e tensão de soldagem, do comprimento do arco e de outros parâmetros pode ser avaliada considerando o circuito elétrico de soldagem e o balanço entre a fusão e a alimentação do eletrodo, juntamente com considerações geométricas [17]:

$\frac{d I}{d t}=\frac{1}{L}\left(U_{\text {Fonte }}-\sum U_{i}\right), \mathrm{e}$

$\frac{d l_{a}}{d t}=w-f$

Onde $U_{\text {Fonte }}$ é a tensão da fonte, $\Sigma_{i}$ é a soma das diferentes quedas de tensão ao longo do circuito de soldagem (incluindo, por exemplo, as quedas de tensão no eletrodo, no contato entre o eletrodo e o bico e no arco), $w$ e $f$ são, respectivamente, as velocidades de fusão e de alimentação do eletrodo, $L$ é a sua indutância, $I$ é a corrente de soldagem e $l_{a}$ é o comprimento do arco. Entre as quedas de tensão, $U_{i}$, duas são particularmente importantes: a queda ao longo do eletrodo $\left(U_{e}\right)$ e a do arco elétrico $\left(U_{a}\right)$.

Com este enfoque, $\mathrm{Xu}$ et al. [18] desenvolveram um modelo que simula o comportamento operacional do processo MIG/MAG. Este modelo permite prever a variação de tensão e corrente, a frequência de curto-circuito e a transição da transferência metálica por curto-circuito para voo livre. Seus resultados não simularam bem a frequência de curto-circuito, nem os valores mínimos e máximos de corrente e tensão com este modo de transferência. Choi, B. et al. [19] apresentaram um modelo matemático similar que incorpora o crescimento e o destacamento da gota na ponta do eletrodo utilizando o modelo de balanço de forças e a teoria de instabilidade de Pinch. Os resultados apresentados indicam valores de frequência e tempo de curto-circuito similares aos de resultados experimentais. Porém, os valores máximos e mínimos de corrente e tensão simulados apresentam maior diferença em relação aos experimentais, principalmente os referentes à corrente. Terasaki e Simpson [20] desenvolveram um modelo similar que, assim como os outros já citados, não prevê o aparecimento de curtoscircuitos de curta duração embora pareça apresentar uma menor diferença nos valores de frequência de curto-circuito simulados e experimentais em comparação com os resultados dos modelos anteriores.

O presente trabalho apresenta o desenvolvimento de um programa de computador que implementa um modelo baseado nas equações (1) e (2) e similar aos apresentados nos trabalhos citados no parágrafo acima. Este modelo apresenta opções para simular alguns aspectos particulares do processo não considerados ainda nos modelos anteriores.

\section{Fundamentos}

O modelo usado neste trabalho foi inicialmente desenvolvido por Modenesi [21] e aperfeiçoado por Santana [22]. Ele se baseia nas equações (1) e (2) e considera as seguintes quedas de tensão ao longo circuito elétrico de soldagem (equação 1 e figura 1):

- $\mathrm{U}_{\text {Parasita: }}$ : Diferença de potencial causada por resistências associadas com cabos e conexões. O seu valor padrão no programa é zero, mas este pode ser alterado pelo usuário e variar durante a simulação de acordo com padrões definidos (senoidal, degrau, etc.) ou de forma aleatória.

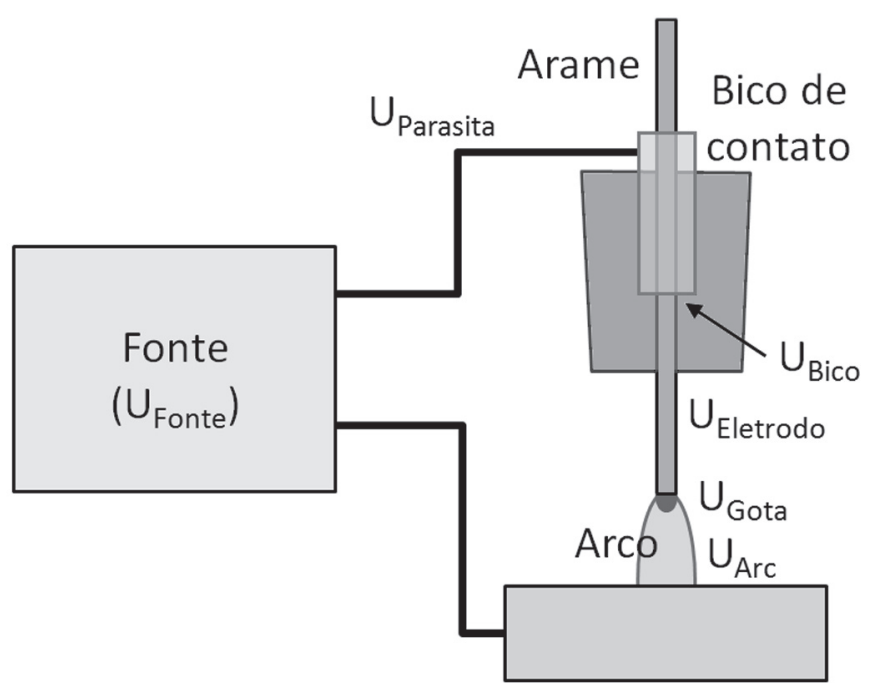

Figura 1. Quedas de tensão elétrica ao longo do circuito de soldagem.

- $\mathrm{U}_{\text {Bico: }}$ : Causada pela resistência entre o bico de contato e o eletrodo.

- $\mathrm{U}_{\text {Eletrodo }}$ : Associada com a resistência elétrica do eletrodo (isto é, a parte do arame por onde a corrente elétrica passa durante a soldagem) e é definida pelo valor da resistividade do arame $\left(\rho_{\mathrm{e}}\right)$. Durante uma operação de soldagem, a resistividade depende do material do eletrodo e da distribuição 
de temperatura ao longo deste. No programa desenvolvido, o valor da resistividade pode depender da corrente de soldagem e do comprimento do eletrodo (s).

- $\mathrm{U}_{\text {Gota }}$ : Associada com a resistência elétrica da gota de metal líquido formada na ponta do eletrodo e com processos ocorrendo entre esta e o arco. Tem uma relação complexa que depende do tamanho da gota e da composição do gás de proteção [20, 23]. Na versão atual do programa, esta é mantida constante e, como padrão, igual a zero.

- $\mathrm{U}_{\mathrm{Arc}}$ : A queda de tensão no arco é modelada no programa como a soma das quedas de tensão nas transições com os eletrodos $\left(\mathrm{U}_{\mathrm{AC}}\right)$ e ao longo da coluna do arco $\left(\mathrm{U}_{\mathrm{COL}}\right)$. Esta última é considerada igual ao produto do comprimento do $\operatorname{arco}\left(\mathrm{L}_{\mathrm{a}}\right)$ pelo módulo do campo elétrico (E) na coluna. O programa permite considerar tanto $\mathrm{U}_{\mathrm{AC}}$ e $\mathrm{E}$ como dependentes da corrente elétrica e do comprimento ao arco. Alternativamente, a variação de tensão ao longo da coluna pode ser representada pela equação empírica obtida por Xu e colaboradores [18]:

$$
\begin{aligned}
& \mathrm{U}_{\mathrm{COL}}=118,6\left\{\exp \left[-0,0857\left(L_{a}+1,054\right)\right]\right. \\
& -1\}\{\exp [-0,000487(I+349,2)]-1\}
\end{aligned}
$$

A tensão fornecida pelo equipamento de soldagem $\left(U_{\text {Fonte }}\right)$ é modelada no programa por uma ou duas retas $\left(\mathrm{U}=\mathrm{U}_{0}-\mathrm{m} \mathrm{I}\right)$. No segundo caso, as retas podem ser usadas simultaneamente ou de forma alternada (em intervalos de tempo definidos pelo usuário). Quando elas são usadas simultaneamente, a seleção da reta a ser usada em um dado instante pode ser feita pelo programa (quando as retas se interceptam) ou pode ser definida arbitrariamente por um valor de tensão de soldagem. Modelos mais sofisticados de fontes poderão ser implementadas em versões futuras do programa.

A indutância (L) do circuito de soldagem pode assumir valores distintos durante as condições de operação com arco e sem arco (em curto-circuito).

$\mathrm{Na}$ equação (2), a velocidade de fusão do eletrodo (w) é modelada pela equação clássica [24] para as condições de soldagem com arco modificada para incluir o efeito da inércia do processo:

$$
w=\alpha I+\beta s(I+\Delta I)^{2}
$$

Onde s é o comprimento do eletrodo e $\alpha$ e $\beta$ podem ser definidos pelo usuário ou calculados pelo programa como [25]:

$\alpha=\frac{U_{A r \varepsilon}}{\gamma A \Delta H_{\text {Tot }}} \quad$ e $\beta=\frac{\rho_{\varepsilon}}{\gamma A^{2} \Delta H_{T o t}}$

onde $\mathrm{U}_{\mathrm{Arc}}$ é a tensão associada com o aquecimento do eletrodo pelo arco, $\gamma$ é a densidade do material do eletrodo, A é a sua seção transversal e $\Delta \mathrm{H}_{\mathrm{Tot}}$ é o calor contido no metal líquido e, eventualmente, vaporizado deixando a ponta quente do eletrodo. Estes valores devem ser fornecidos pelo usuário.

Durante a ocorrência de um curto circuito, a equação (4) é simplificada para:

$$
w=\beta s(I+\Delta I)^{2}
$$

O termo $\Delta \mathrm{I}$ foi incluído para levar em consideração a influência de uma inércia térmica no aquecimento do eletrodo e, por consequência, em seus efeitos, particularmente na fusão do eletrodo por efeito Joule. Para reproduzir este efeito, Simpson [20] usou como tempo de atraso, o necessário para um dado ponto do arame percorrer uma distância igual à metade do comprimento do eletrodo, $t_{\text {Lag }}=s /(2 f)$, onde $f$ é a velocidade de alimentação. No programa aqui apresentado, este efeito foi simulado pela introdução de um termo $\Delta \mathrm{I}$ que é somado ao valor instantâneo da corrente e que leva em consideração a influência de valores anteriormente calculados da corrente de soldagem. Este termo foi definido através de uma expressão recorrente da forma

$$
\begin{aligned}
& \Delta I_{i}=I_{i}-\left(I_{\text {Lag }}\right)_{i} \text { e } \quad\left(I_{\text {Lag }}\right)_{i}= \\
& {\left[K_{\text {Lag }} \cdot I_{i}+\left(1-K_{\text {Lag }}\right) \cdot\left(I_{\text {Lag }}\right)_{i-1}\right]}
\end{aligned}
$$

$\mathrm{O}$ coeficiente $\mathrm{K}_{\text {Lag }}$ depende de um tempo de atraso $\left(\mathrm{t}_{\text {Lag }}\right)$ definido pelo usuário e é calculado como:

$$
K_{\text {Lag }}=1-\exp \left(-2,302585 \cdot \frac{\Delta t}{t_{\text {Lag }}}\right)
$$

onde $\Delta$ t é o passo de integração usado.

A velocidade de alimentação do arame é selecionada pelo usuário e mantida fixa durante a simulação. Adicionalmente, perturbações periódicas (senoidal, em degrau, etc.) ou aleatórias na velocidade de alimentação do arame podem ser selecionadas.

Além da perturbação na velocidade de alimentação, o programa considera a possibilidade de perturbações similares no comprimento do arco (devido, por exemplo, a oscilações do metal líquido na ponta do eletrodo e na poça de fusão) e na distância do bico de contato à peça. Além disto, o programa permite atrasar final de períodos de curto-circuito admitindo a formação de uma ponte de metal líquido entre a ponta do arame e a poça de fusão e permite a ocorrência de períodos de picos de tensão anormal $[26,27]$ aleatoriamente em operação com arco ou ao final de períodos de curto-circuito.

\subsection{Desenvolvimento do programa}

O programa foi desenvolvido em linguagem $\mathrm{C}++$, sendo organizado em torno de quatro objetos básicos (Arco, Eletrodo, Fonte e Processo). Os três primeiros incorporam as propriedades e características dos três elementos do processo de soldagem. $\mathrm{O}$ quarto objeto contem propriedades e características gerais e comuns do processo e permite a ligação entre Arco, Eletrodo e Fonte.

As equações (1) e (2) são resolvidas numericamente pelo método de Runge-Kunta de $3^{\mathrm{a}}$ ordem (podendo, também, ser usado o método mais simples de Euler). Para fazer uma simulação, o usuário define as condições de soldagem, os valores das propriedades dos materiais e do processo e a ocorrência de perturbações. Além disto, ele deve escolher o passo de avanço da solução e o intervalo total de tempo (estes dois parâmetros definem o número total de pontos que serão calculados e, desta forma, o tempo de processamento e a quantidade de memória do computador que será usada). 
Tabela 1. Valores usuais de alguns parâmetros utilizados nas simulações.

\begin{tabular}{l|c|l|l|c|l}
\hline \multicolumn{1}{c|}{ Parâmetro } & Valor & \multicolumn{1}{c|}{ Unidades } & \multicolumn{1}{c|}{ Parâmetro } & Valor & \multicolumn{1}{c}{ Unidades } \\
\hline Diâmetro do arame & 1,2 & $\mathrm{~mm}$ & $\mathrm{DBCP}(*)$ & 16 & $\mathrm{~mm}$ \\
\hline Resistividade & 0,6 & $\mu \Omega . \mathrm{m}$ & Resistência bico-arame & 0,0075 & $\Omega$ \\
\hline Alfa & 0,27 & $\mathrm{~mm} / \mathrm{A} \mathrm{s}$ & Beta & $5,9 \times 10^{-5}$ & $1 /\left(\mathrm{s} \mathrm{A}^{2}\right)$ \\
\hline Inclinação da fonte & 0,04 & $\mathrm{~V} / \mathrm{A}$ & Indutância & $2 \times 10^{-4}$ & $\mathrm{H}$ \\
\hline $\mathrm{E}$ (gás rico em Ar) & 750 & $\mathrm{~V} / \mathrm{m}$ & $\mathrm{E}\left(\mathrm{CO}_{2}\right)$ & 1800 & $\mathrm{~V} / \mathrm{m}$ \\
\hline $\mathrm{U}_{\mathrm{AC}}$ (gás rico em Ar) & 17 & $\mathrm{~V}$ & $\mathrm{U}_{\mathrm{AC}}\left(\mathrm{CO}_{2}\right)$ & 22 & $\mathrm{~V}$ \\
\hline
\end{tabular}

(*) Distância do bico de contato à peça.

Ao final do processamento, o programa mostra os resultados na forma de gráficos de corrente, tensão de soldagem e comprimento do arco em função do tempo e os valores médios destes parâmetros no último quarto da simulação. Os resultados podem ser salvos para análise posterior em um programa específico. As condições de simulação também podem ser salvas para serem usadas e modificadas posteriormente em outra simulação.

\section{Resultados e Discussão}

Este item apresenta resultados de algumas simulações e os compara com alguns resultados de testes reais. Não se procurou reproduzir os valores exatos de corrente e tensão dos testes reais, mas comparar as formas dos sinais obtidos e as tendências gerais observadas na operação real do processo MIG/MAG e as previstas pela implementação do modelo. Exceto quando explicitado, os valores adotados dos parâmetros do modelo são os mostrados na tabela 1. Estes valores são baseados em resultados de testes experimentais $[17,22] \mathrm{e}$ em dados da literatura [18-20].

\subsection{Soldagem convencional com transferência por queda livre}

A figura 2 ilustra a simulação de condições de soldagem sem curtos-circuitos com velocidade de alimentação do arame (f) igual a $4 \mathrm{~m} / \mathrm{min}$ em que foram feitas variações instantâneas de $3 \mathrm{~mm}$ no comprimento do arco, em um caso sem e no outro com alteração correspondente na DBCP. As variações de corrente e tensão são as esperadas e, no caso em que a distância do bico de contato à peça não é alterado, as condições originais de operação são restauradas ao final do intervalo de tempo mostrado. Para o segundo caso (com aumento de $3 \mathrm{~mm}$ na DBCP, as condições operacionais se estabilizam com um menor valor de corrente.

Em condições reais de soldagem com arco aberto, os valores de corrente e tensão sofrem flutuações associadas com vários fenômenos que ocorrem no processo, particularmente com a transferência de metal de adição entre o eletrodo e a poça de fusão. $\mathrm{O}$ modelo aqui apresentado pode ser modificado para incorporar alguns aspectos do crescimento e destacamento da gota de metal na ponta do eletrodo [20]. Na versão atual do programa aqui descrito, estes aspectos, baseados parcialmente em considerações teóricas, não foram ainda implementados. Algumas características da transferência de metal globular

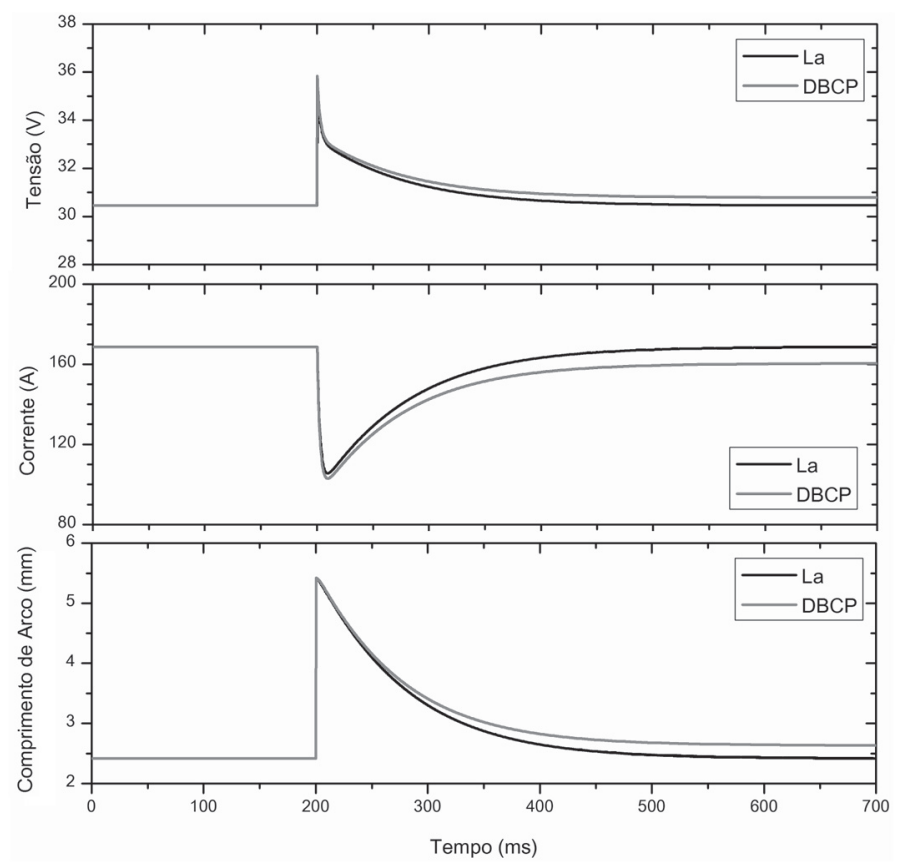

Figura 2. Simulação da soldagem MIG/MAG usando $\mathrm{CO}_{2}$ como proteção com a imposição de uma variação instantânea

no comprimento do arco sem e com alteração da DBCP.

podem ser, contudo, simuladas através colocação de perturbações no comprimento do arco. A figura 3 compara as variações de tensão e corrente de um teste de soldagem MIG/MAG com proteção de $\mathrm{CO}_{2}$ e com transferência globular. Nos resultados do teste real podem ser observados três eventos, com variações bruscas da tensão e corrente correspondentes ao destacamento da gota, seguidos por períodos com uma mudança suave dessas associadas com o crescimento da gota na ponta do eletrodo. Neste período, são observadas ainda flutuações possivelmente causadas por oscilações na gota de metal líquido e na poça de fusão. Na simulação este comportamento foi reproduzido pela imposição de tipos de variações periódicas ao comprimento do arco: (a) variações bruscas (tipo "dente de serra") para reproduzir o destacamento da gota e (b) variações senoidais de maior frequência para reproduzir as oscilações do metal líquido.

Para a soldagem com proteção de $\mathrm{CO}_{2}$ puro, a variação que foi imposta ao comprimento do arco permite emular, de forma satisfatória, as variações observadas na tensão e corrente de soldagem associadas com a transferência de metal. Contudo, em misturas com um teor mais elevado de argônio, ocorre uma 
inversão nas mudanças mostradas [23], cuja origem não é ainda bem conhecida. Trabalhando com um modelo similar ao descrito neste trabalho, Simpson [29] reproduziu este comportamento de forma empírica considerando a existência de uma diferença de potencial na superfície da gota descrita por:

$U_{S G}=\varphi \frac{V_{\text {Gota }}}{d_{w}^{2}} I$

onde $\varphi$ é uma constante, $\mathrm{V}_{\text {Gota }}$ é o volume da gota na ponta do eletrodo e $\mathrm{d}_{\mathrm{w}}$ é o seu diâmetro. Neste caso, a mudança de comportamento citada era reproduzida simplesmente mudando o sinal do termo $\varphi$ na equação (10).

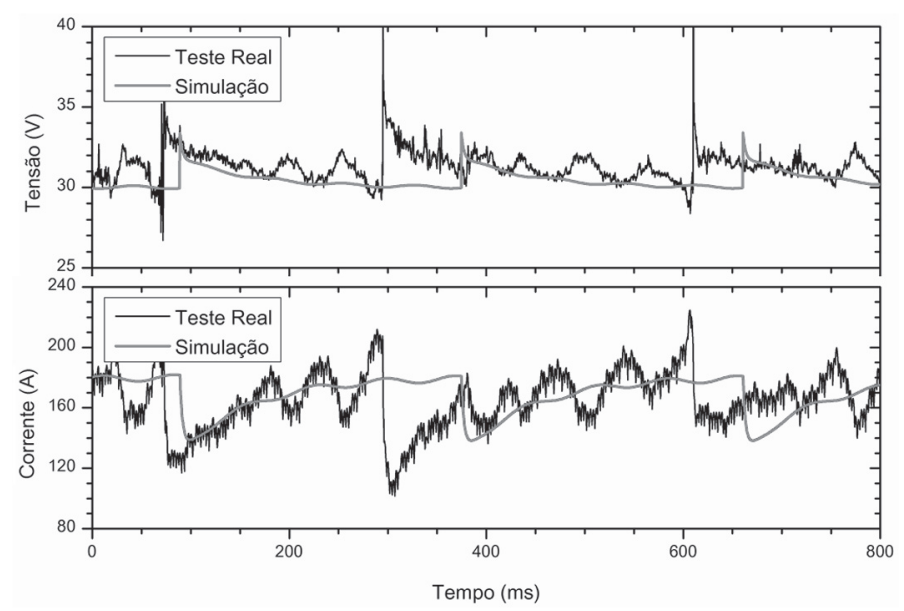

Figura 3. Comparação entre um teste de soldagem MIG/ MAG com proteção de $\mathrm{CO}_{2}$, velocidade de alimentação de $4 \mathrm{~m} /$ min e transferência globular com o resultado de uma simulação correspondente.

\subsection{Soldagem com transferência por curto-circuito}

Em operação com curto-circuito, o processo opera em duas condições distintas (em arco e em curto) e alternadas. O modelo considera o início da ocorrência de um curto circuito quando o comprimento de arco se iguala a zero $\left(\mathrm{L}_{\mathrm{a}} \rightarrow 0\right)$. O término deste ocorre quando a ponta do arame se afasta da superfície da peça de uma distância especifica maior ou igual a zero.

A figura 4 ilustra o resultado de uma simulação simples com transferência por curto-circuito. Ela reproduz alguns aspectos típicos observados em condições reais de soldagem, particularmente a alternância de períodos de operação em arco e em curto-circuito com as variações esperadas de corrente e tensão. Por outro lado, e, como esperado, a simulação não é capaz de reproduzir o aspecto aleatório da duração dos intervalos destes períodos e de sua intensidade observado em soldagem. Essas características são afetadas por diversos fatores, incluindo variações nas condições do arco elétrico e flutuações no comprimento do arco devido a oscilações do metal líquido tanto na poça de fusão como na gota ligada ao eletrodo. A figura 5 mostra o efeito da introdução, na simulação, de duas oscilações senoidais simultâneas no comprimento do arco com frequências de 3,5 e $171 \mathrm{~Hz}$ e amplitudes de 0,1 e 0,05 mm. Pode-se observar que os períodos de transferências, mesmo com esta perturbação relativamente simples, passam a apresentar duração variável e com um comportamento aparentemente aleatório.

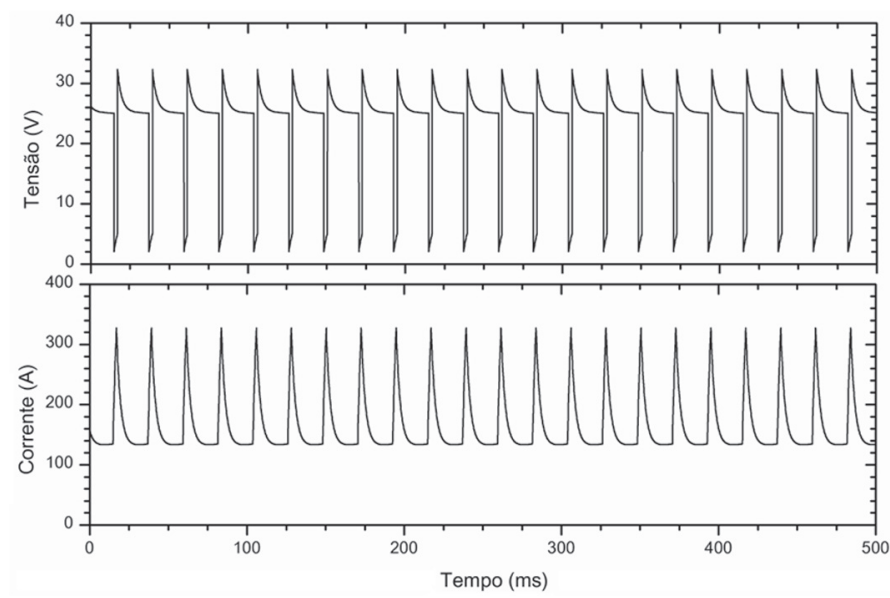

Figura 4. Simulação de uma condição de soldagem MIG/ MAG com proteção de $\mathrm{CO}_{2}$, velocidade de alimentação de $4 \mathrm{~m} /$ min e transferência por curto circuito.

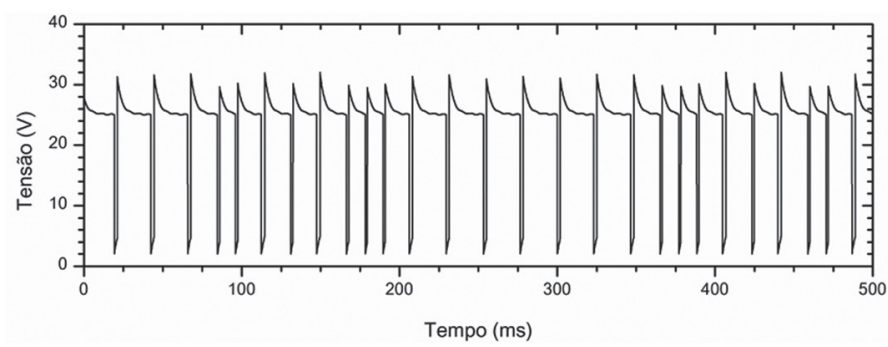

Figura 5. Simulação similar à da figura anterior, mas com a introdução de perturbações senoidais no comprimento do arco com freqüências de 3,5 e $171 \mathrm{~Hz}$ e amplitudes de $0,1 \mathrm{e}$ $0,05 \mathrm{~mm}$.

Além de oscilações no comprimento do arco, o programa permite introduzir outras perturbações como, por exemplo, variações na velocidade de alimentação ou na resistência parasita, a formação de uma ponte entre o eletrodo e a peça ao final do curto circuito (cujo comprimento é definido pelo usuário) e o retardo no efeito da corrente na fusão do eletrodo (equações 4, 7, 8 e 9). A figura 6 mostra a variação da corrente média de soldagem com a tensão em vazio da fonte de soldagem para simulações de condições de soldagem com curto-circuito (valores nas curvas localizados abaixo da seta colocada na figura). Esta figura também reproduz resultados de medidas em testes reais em soldagem (círculos). Estes resultados, em condições de operação com curtos circuitos (entre as duas linhas tracejadas da figura 6) indicam uma tendência da corrente aumentar fracamente com a tensão. Esta tendência é diferente da encontrada na simulação sem perturbação ou na com retardo da corrente, para as quais a corrente diminui com a tensão, e na com imposição de oscilações do arco, para a qual a corrente aumenta de forma mais forte com a tensão.

Estes resultados indicam que as simulações com as perturbações simples usadas não conseguem reproduzir de forma fiel a variação da corrente com a tensão em condições reais com curtos-circuitos. Naturalmente, os fenômenos que 
ocorrem tanto durante os períodos de arco como os de curtocircuito e que influenciam o valor da corrente de soldagem são muito mais complexos que as perturbações introduzidas no modelo. Este ainda deverá ser aperfeiçoado em vários aspectos, mas, possivelmente, uma reprodução fiel de detalhes do processo não será alcançada.

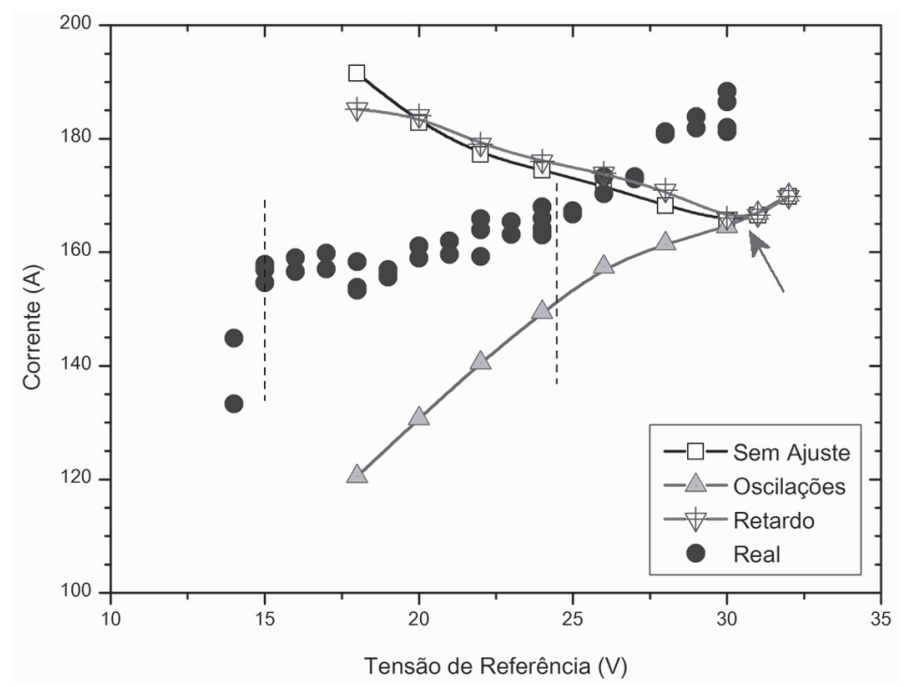

Figura 6. Variação da corrente média de soldagem com a tensão de referência. As curvas com pontos são simulações e os pontos cheios são resultados de soldas reais (proteção: Ar- $25 \% \mathrm{CO}_{2}$ ). Nas simulações, a tensão de referência é a em vazio da fonte, nas soldas reais é a ajustada nesta. Oscilações: Simulação com perturbações senoidais de 3,0 e $151 \mathrm{~Hz}$ e de 0,1 e $0,05 \mathrm{~mm}$ no comprimento do arco. Retardo: Simulação com tempo de retardo de $2 \mathrm{~ms}$ na corrente. Os pontos da soldagem real entre as duas linhas tracejadas são de operação com curtocircuito e sem instabilidade excessiva.

A figura 7 compara resultados de soldas reais e de simulações da frequência média de transferência por curto-circuito.

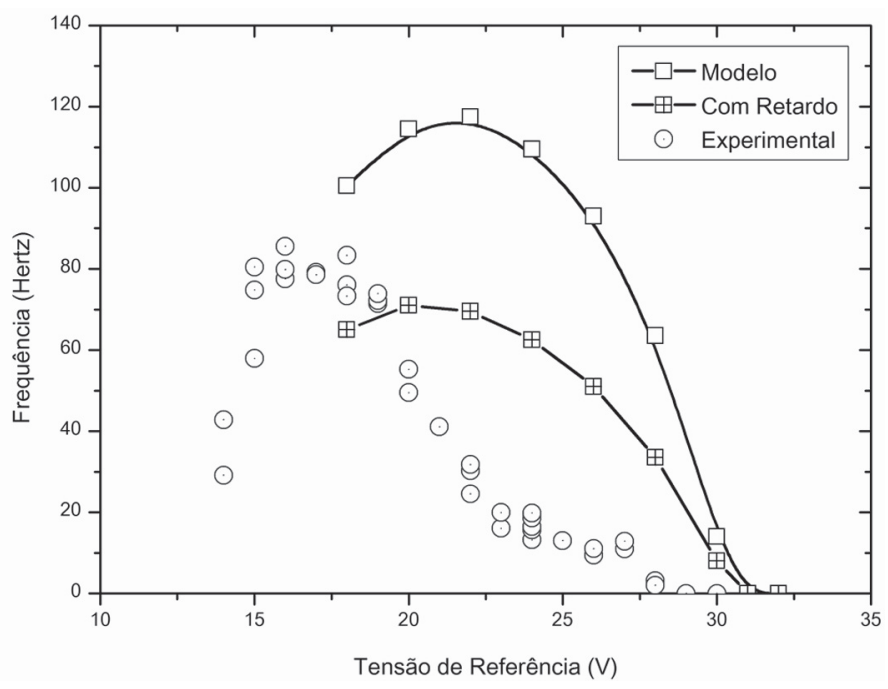

Figura 7. Variação da frequência de transferência por curtocircuito em soldagem com proteção de $\mathrm{Ar}-25 \% \mathrm{CO}_{2}$ (círculos) e em simulações (símbolos + linha).
A frequência de transferência varia com a tensão passando por um máximo próximo do qual o processo opera de forma mais estável, por exemplo, com menor formação de respingos. Para tensões menores, a instabilidade aumenta fortemente, sendo basicamente impossível de usar o processo. Para as tensões maiores, os curtos-circuitos se tornam mais espaçados e o processo eventualmente passa a operar com transferência por queda livre.

As simulações reproduziram este comportamento (figura 7), embora estas não incorporem alguns dos fenômenos responsáveis por este comportamento na soldagem real. No modelo, esta variação decorre do crescimento dos tempos de curto-circuito que passam a controlar o período de transferência, Em soldagem, associada com estes curtos-circuitos mais longos, ocorre a formação de respingos formados por pedaços do arame que ocasionam forte variação da distância entre a ponta do eletrodo e a peça e mudanças nas condições de transferência.

Além deste aspecto, existem diferenças adicionais entre as curvas simuladas e a experimental da figura 7. Em particular, pode-se observar, no caso da soldagem real com tensões mais elevadas, uma faixa de tensões em que a frequência de transferência se mantém baixa e varia pouco. Esta faixa deve estar associada com um controle da formação dos curtos circuitos por variações relativamente grandes no comprimento do arco pelas oscilações da gota na ponta do eletrodo e da poça de fusão.

\subsection{Soldagem com corrente pulsada}

A figura 8 compara as variações de corrente e tensão de uma soldagem com corrente pulsada e de uma simulação. Esta foi feita considerando uma fonte com imposição de corrente tanto no pulso como na base usando os tempos e os valores de corrente da soldagem real. O oscilograma simulado da tensão de soldagem reproduz o formato geral do oscilograma da solda real exceto por variações instantâneas, possivelmente associadas com a transferência de metal, e o decaimento da tensão durante o período de base. Este último está possivelmente associado com variações do comprimento de arco devido ao destacamento da gota e às suas oscilações e da poça de fusão, o que não foi considerado na simulação.

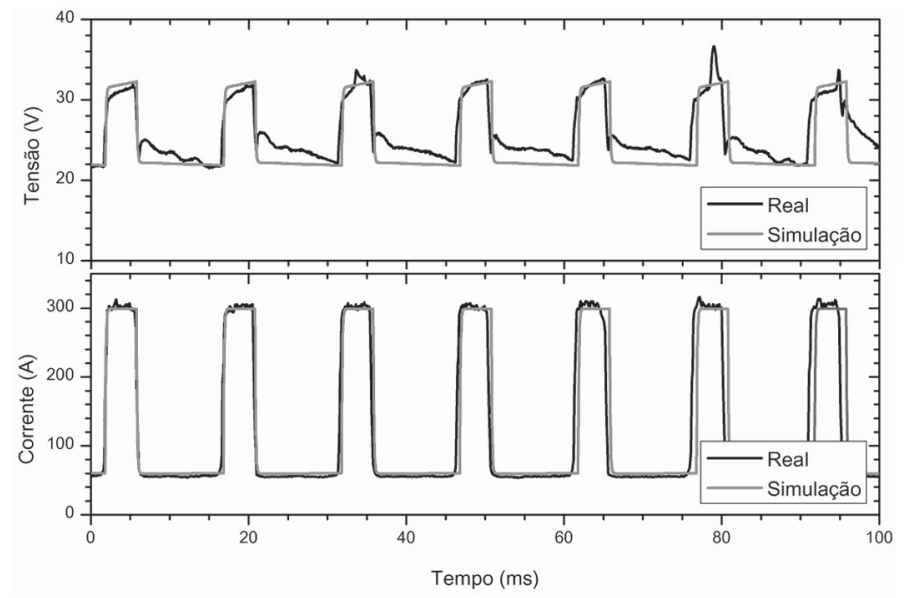

Figura 8. Oscilogramas de uma solda real com corrente pulsada com proteção de $\mathrm{Ar}-2 \% \mathrm{O}_{2}$ e de uma simulação. 


\section{Conclusões}

Este trabalho apresenta o desenvolvimento de um programa de computador que usa um modelo matemático para simular as variações de corrente e tensão e de comprimento do arco durante a soldagem MIG/MAG. As suas principais conclusões foram:

- O modelo desenvolvido permite simular o comportamento elétrico e as variações de comprimento do arco durante a soldagem MIG/MAG em diferentes situações, incluindo a soldagem convencional com transferência por queda livre, a soldagem com transferência por curto-circuito e a soldagem com corrente pulsada.

- A variabilidade aparentemente aleatória dos períodos de transferência durante a soldagem com curtos-circuitos pode ser simulada pela introdução de perturbações simples, como, por exemplo, oscilações periódicas do comprimento do arco.

- Variações de corrente e tensão associadas com a transferência de metal por queda livre com proteção de $\mathrm{CO}_{2}$ também podem ser simuladas pela introdução no modelo de perturbações no comprimento do arco. Contudo, a mesma abordagem não funciona de forma adequada quando o gás de proteção é rico em argônio devido aos processos que ocorrem durante a transferência e alteram o sinal das variações de tensão.

- O modelo é também capaz de reproduzir as alterações no tempo de transferência com a tensão de operação na soldagem com curtos-circuitos. Na sua forma atual, contudo, este comportamento do modelo não foi associado com fenômenos similares aos que ocorrem em condições reais de soldagem.

\section{Referências Bibliográficas}

[1] KIM, I. S., KWON, W. H., SIORES, E., An investigation of a Mathematical Model for Predicting Weld Bead Geometry, Canadian Metallurgical Quarterly, v. 35, n. 4, p. 385-392, 1996. [2] KIM, I. S., SON, J. S., KIM, I. G., KIM, J. Y., KIM, O. S., A study on relationship between process variables and bead penetration for robotic $\mathrm{CO}_{2}$ arc welding, Journal of Materials Processing Technology, n. 136, p.139-145, 2003.

[3] MURUGANA N., GUNARAJ, V., Prediction and control of weld bead geometry and shape relationships in submerged arc welding of pipes, Journal of Materials Processing Technology, n. 168, p. 478-487, 2005.

[4] QIANG, Z., LIGONG, C., JIANNING, X., CHUNZHEN, N., DEFU, H., Modeling and estimating for external characteristic of welding power source, Int. J. Adv. Manuf. Technol., v. 29, p. 269-272, 2006 DOI 10.1007/s00170-005-2516-1.

[5] PALANI, P.K., MURUGAN, N., Optimization of weld bead geometry for stainless steel claddings deposited by FCAW, Journal of Materials Processing Technology, n. 190, p.291-299, 2007.

[6] JUANG, S.C., TARNG, Y.S., LII, H.R., A comparison between the back-propagation and counter-propagation networks in the modeling of the TIG welding process, Journal of Materials Processing Technology, n. 75, p. 54-62, 1998.

[7] GWAN-HYUNG KIM, SUNG-IN KANG, SANG-BAE LEE, A Study of the Estimate of Weld Bead Shape and the Compensation of Welding parameters by Considering Weld
Defects in Horizontal Fillet Welding, In: Third International Conference on Knowledge-Based Intelligent Information Engineeing Systems, 31th Aug-1st Sept 1999, Adelaide, Australia p. 212-216.

[8] DUCHARME, R., KAPADIA, P., DOWDEN, J., THORTON, R., RICHARDSON, I., A mathematical model of the arc in electric arc welding including shielding gas flow and cathode spot location, J. Phys. D: Appl. Phys., v. 28, p. 1840-1850, 1995. [9] GOODARZI, M., CHOO, R., TAKASU, T., TOGURI, J.M., The effect of the cathode tip angle on the gas tungsten arc welding arc and weld pool: II. The mathematical model for the weld pool, J. Phys. D: Appl. Phys., v. 31, p. 569-583, 1998.

[10] WANG, F., HOU, W.K., HU S.J., KANNATEY-ASIBU, E., SCHULTZ, W.W., WANG, P.C.: Modelling and analysis of metal transfer in gas metal arc welding, J. Phys. D Appl. Phys., v. 36, p. 1143-1152, 2003.

[11] ZHAO, P.C. et al., Numerical simulation of the dynamic characteristics of weld pool geometry with step-changes of welding parameters, Modelling Simul. Mater. Sci. Eng., v. 12, p. 765-780, 2004.

[12] WENDELSTORF, J., Ab initio modelling of thermal plasma gas discharges (electric arcs), PhD Thesis: Technischen Universität Carolo-Wilhelmina 145p., 2000.

[13] QUINN, T.P., SZANTO, M., GILAD, I., SHAI, I., Coupled arc and droplet model of GMAW, Science and Technology of Welding and Joining, v. 10, n. 1, p. 113-119, 2005.

[14] CHO, M.H., FARSON, D.F., Understanding Bead Hump Formation in Gas Metal Arc Welding Using a Numerical Simulation, Metallurgical and Materials Transactions B, v. 38B, p. 305-319, 2007.

[15] KUMAR, A., ZHANG, W., DEBROY, T., Improving reliability of modelling heat and fluid flow in complex gas metal arc fillet welds - part I: an engineering physics model, J. Phys. D: Appl. Phys., v. 38, p. 119-126, 2005.

[16] TAYLOR, G.A., HUGHES, M., STRUSEVICH, N., PERICLEOUS, K., The Application of Three Dimensional Finite Volume Methods to the Modelling of Welding Phenomena, In: Modeling of Casting, Welding and Advanced Solidification Processes IX, Editado por Sahm, P.R., Hansen, P.N. e Conley, J.G., 2000, 8p.

[17] MODENESI, P. J., COSTA, M. C. M. S., SANTANA, I. J., BERGANHOLI, J. P. P., Estudo de alguns parâmetros básicos da soldagem GMAW com transferência por curto-circuito, Soldagem \& Inspeção, v. 16, n. 1, p.12-21, 2011.

[18] XU, P.; RADOS, M.; SIMPSON, S. W., Circuit simulation for gas metal arc welding. Science and Technology of welding and Joining, p. 341-346, 1999.

[19] CHOI, B. J. H.; LEE, J. Y.; YOO, C. D., Simulation of dynamic behavior in a GMAW system, Welding Journal, N. 10, p. 239-245, 2001.

[20] TERASAKI, H.; SIMPSON, S. W., Circuit simulation for gas metal arc welding system, In: The $47^{\text {th }}$ International Midwest Symposium on Circuits and System. University of Sydney. p. 387-390, 2006.

[21] MODENESI, P. J. Evolução da Temperatura do Bico de Contato na Soldagem MIG/MAG, In: XXIII Encontro Nacional de Tecnologia da Soldagem, 1997, São Paulo, Associação 
Brasileira de Soldagem, p. 215-223, 1997.

[22] SANTANA, I. J. Modelamento Matemático do Processo de Soldagem GMAW, Tese, Universidade Federal de Minas Gerais, 2010, 153p.

[23] SCOTTI, A., PONOMAREV, V., RESENDE, A.: The influence of electrode material and shielding gas mixture on the specific electric resistances of the drop/column of the arc in MIG/MAG welding - IIW Doc. XII-1909-06 9p. 2007.

[24] QUINTINO, L., ALLUM, C. J. Pulsed GMAW: interactions between process parameters-Part 1/2. Weld. \& Metal Fab., Março/Abril, pp. 85-89, 126-129, 1984.

[25] MODENESI, P. J. Introdução à Física do Arco Elétrico e sua Aplicação à Soldagem dos Metais, UFMG, 162p., 2011. Extraído em julho de 2011 de: http://www.demet.ufmg.br/labs/ soldagem/textos/fisica da soldagem.pdf

[26] MODENESI, P.J.; NIXON, J.H. Arc instability phenomena in GMA welding. Welding Journal, v.73, n.9, p.219s-224s, 1994.

[27] TONG, H. et al. Observations of the phenomenon of abnormal arc voltage occurring in pulsed metal inert gas welding of aluminium alloy. Science and Technology of Welding and Joining, v.10, n.6, p. 695-700, 2005.

[28] SIMPSON, S. W.: Metal transfer instability in gas metal arc welding, Science and Technology of Welding and Joining, v. 14, n. 4 p. 262-273, 2009. 